Article

\title{
Ontology-Based Representation for Accessible OpenCourseWare Systems ${ }^{\dagger}$
}

\author{
Mirette Elias ${ }^{1,2, *}$, Steffen Lohmann ${ }^{2}$ and Sören Auer ${ }^{3}$ \\ 1 Institute of Computer Science, University of Bonn, 53115 Bonn, Germany \\ 2 Fraunhofer IAIS, 53757 St. Augustin, Germany; steffen.lohmann@iais.fraunhofer.de \\ 3 Technische Informationsbibliothek (TIB), 30167 Hannover, Germany; soeren.auer@tib.eu \\ * Correspondence: melias@uni-bonn.de \\ $+\quad$ This paper is an extended version of our paper published in the Proceedings of the 8th International \\ Conference on Knowledge Engineering and Semantic Web (KESW 2017).
}

Received: 29 October 2018; Accepted: 26 November 2018; Published: 29 November 2018

\begin{abstract}
OpenCourseWare (OCW) systems have been established to provide open educational resources that are accessible by anyone, including learners with special accessibility needs and preferences. We need to find a formal and interoperable way to describe these preferences in order to use them in OCW systems and retrieve relevant educational resources. This formal representation should use standard accessibility definitions of OCW that can be reused by other OCW systems to represent accessibility concepts. In this article, we present an ontology to represent the accessibility needs of learners with respect to the IMS AfA specifications. The ontology definitions together with rule-based queries are used to retrieve relevant educational resources. Related to this, we developed a user interface component that enables users to create accessibility profiles representing their individual needs and preferences based on our ontology. We evaluated the approach with five examples profiles.
\end{abstract}

Keywords: OpenCourseWare; OCW; ACCESSIBLE; IMS AfA; ontologies; web accessibility

\section{Introduction}

OpenCourseWare (OCW) systems provide a means for distributing free educational content to a wide range of learners over the web [1]. These learners include people with disabilities who have diverse needs, in terms of type and severity of their disabilities, which should be addressed by OCW systems that aim to be inclusive. Designing one system that meets the needs of all learners is usually not possible, as learners have different needs and preferences, in particular disabled learners. In a recent survey we conducted [2], we found that there is still no comprehensive formal representation of learners with disabilities and their particular accessibility needs in e-learning contexts.

This article is an extension to our previous work [3], where the main objective of the research was to define a way to represent the needs and preferences of learners, the properties of educational materials, and the conditions to map them to each other. In that work, we created an ontology, which we call AccessibleOCW, to represent these accessibility needs in order to structure the knowledge and to access the information for recommendations and adaptations in e-learning contexts, with the goal to help in the process of generating a personalized OCW experience. We reused and extended concepts of the ACCESSIBLE ontology [4] to represent users with disabilities along with the accessibility specifications of e-learning systems, as defined by the specification IMS Global Access for All (AfA) [5]. We decided to use the IMS AfA concepts in our ontology because their specifications are adopted by the ISO/IEC 24751 and their framework is based on the W3C Web Content Accessibility Guidelines 
(WCAG 2.0), thus ensuring the reliability and validity of our concepts and encouraging the reuse of our ontology.

In this article, we are extending and evaluating our ontology by defining several learner profiles. In particular, we are representing these example profiles with the recommended properties as per the user definition of the Global Public Inclusive Infrastructure (GPII) [6]. These profiles are then used to populate a user interface where learners can easily select and edit their preferences. One benefit of using an ontology is the possibility to reuse and extend its concepts in other applications; in our case, in the context of e-learning and OCW in particular. As we are making use of the IMS AfA specification to build our AccessibleOCW ontology, the ontology can be reused as a schema for representing the main concepts of OCW in other systems.

The remainder of this article is organized as follows: Section 2 describes the approach we used to design our solution; Section 3 briefly introduces the ontology concepts; Section 4 represents five learner profiles realized with our ontology; Section 5 explains the implementation of the learner profile web form; Section 6 evaluates the approach and profiles; Section 7 reviews the related work to our proposed solution; finally, Section 8 concludes our work and suggests future research.

\section{An Accessible OpenCourseWare Approach}

Our research is conducted in the context of the SlideWiki project (https:/ / slidewiki.eu), which is concerned with the development of an accessible OpenCourseWare system making use of semantic technologies. A main goal of the envisioned OCW system is that it can be adapted to the needs and preferences of various learners. In order to achieve this goal using semantic technologies, we split our approach into two main phases: (i) formalizing OCW concepts in an ontology and (ii) implementing a personalization module.

\subsection{Formalizing OCW Concepts in an Ontology}

This phase focused on formalizing the learners' needs and preferences, together with the properties of the educational resources, in an ontology in order to provide a standard representation and allow reuse of these concepts. In our previous research [2], we reviewed the state of the art and selected the most relevant specifications and guidelines to built our AccessibleOCW ontology [3]. The ontology provides a structured schema for defining the metadata of the learner's profile and the educational resources.

The Learner concept, which defines an individual learner's needs and preferences, is based on two aspects: (i) the properties of each type of disability (blind, deaf, etc.); and (ii) the special accessibility needs of e-learning systems (e.g., education level, complexity level, and preferred language for the learning resources). Each type of disability has specific characteristics, functional limitations, and capabilities as per the medical standard definition of the International Classification of Functioning, Disability and Health (ICF) [7]. This domain knowledge of defining disabilities with their properties is formalized in the ACCESSIBLE ontology [4], which represents the disability types, characteristics, and assistive technologies as per the ICF standards. We reused the User concept of the ACCESSIBLE ontology to extend our Learner concept with this domain knowledge. For accessibility needs related to e-learning, we formalized the concepts of the specification IMS AfA Personal Needs \& Preferences (PNP) [8] in our ontology.

The Digital Resource concept refers to any type of educational material (e.g., video, presentation, text document) in an OCW system. We use the IMS AfA Digital Resource Description (DRD) specification [9] to define the properties of the digital resource concept. The DRD properties were already materialized as metadata in schema.org [10], so formalizing these properties into our ontology would allow for the reuse of any materials that are tagged with the accessibility metadata at schema.org (https://schema.org/accessibilityFeature). 


\subsection{Implementing a Personalization Module}

The personalization module uses the knowledge of the AccessibleOCW ontology to store the learner profiles and educational resources, infer and recommend the most appropriate resources and allow for adaptations of the OCW system in accordance with the learner's input profile. The process model, illustrated in Figure 1, describes the interaction between the learner and the personalization module, and the usage of the AccessibleOCW ontology and data stores. The process sequence is defined in the following steps:

1. Learners are asked to create a profile by initially selecting from five pre-defined accessibility profiles. These profiles with all their properties have been created on the basis of the user definitions of the specifications GPII [6] and IMS PNP [8]. The creation and representation of these profiles, as listed in Figure 2, will be explained in detail in Section 4.

2. The personalization module loads the initial preferences of the selected type of profile as per the definitions of the AccessibleOCW ontology. The learners are free to edit and save these preferences in their profile. Thus, we consider the individual needs and preferences of learners in our work, even if they share the same type of disability.

3. When the learner logs into the OCW system, the personalization module retrieves the educational resources in the most appropriate representation as per the learner's profile. The mappings between the learner's preferences and the educational resources are also defined in the AccessibleOCW ontology, and we use SPARQL queries to retrieve them.

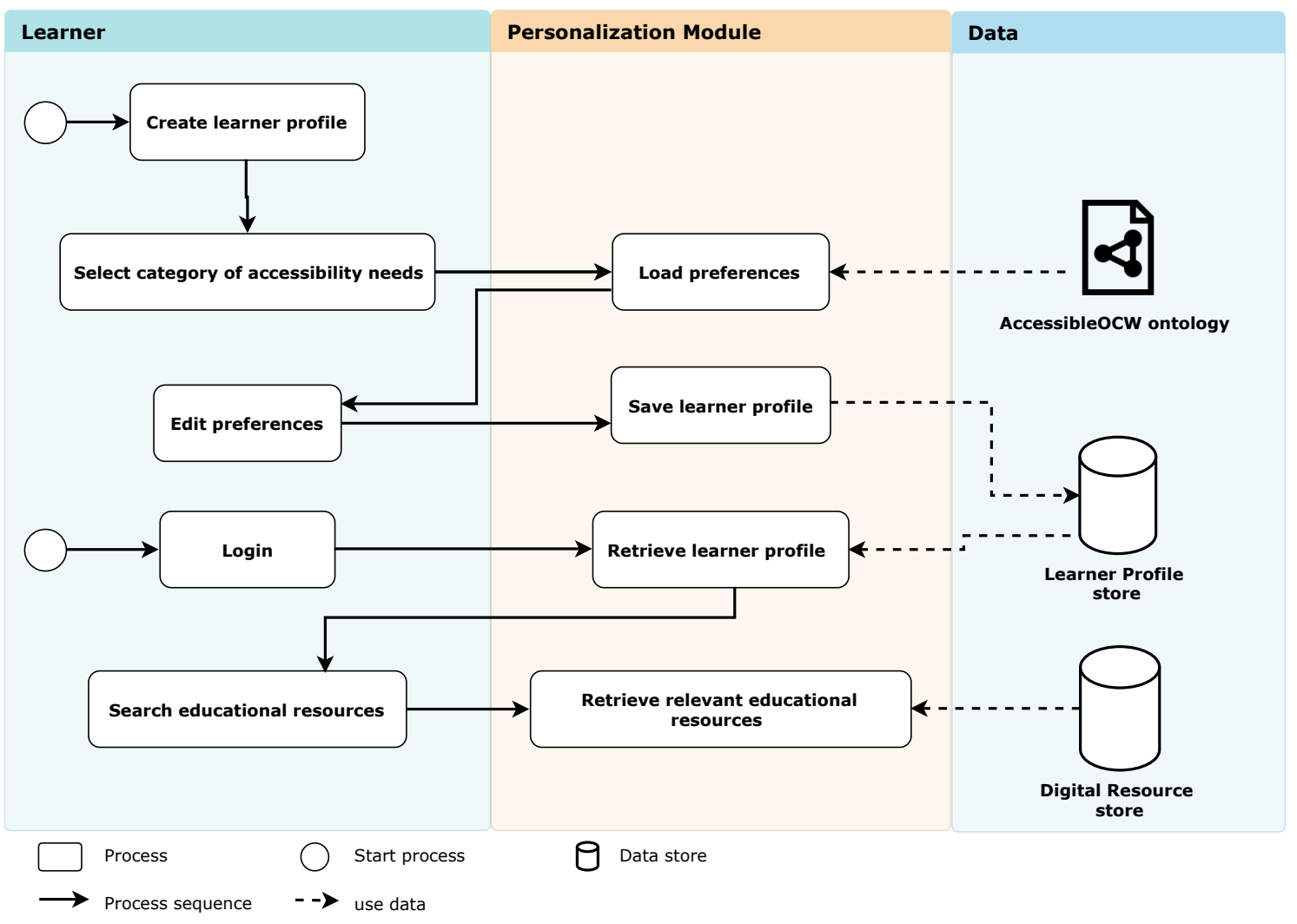

Figure 1. Process model of the proposed solution. 


\begin{tabular}{|c|c|c|c|c|c|c|c|c|c|c|c|c|c|c|c|}
\hline Learner Profiles (GPIII) & \multicolumn{3}{|c|}{ Blindness } & \multicolumn{3}{|c|}{ Low Vision } & \multicolumn{3}{|c|}{ Deaf and Hard of Hearing } & 9 & \multicolumn{2}{|c|}{$\begin{array}{l}\text { Cognitive, Language, and Learning } \\
\text { Disabilities }\end{array}$} & \multicolumn{3}{|c|}{ Physical Disabilities } \\
\hline \multicolumn{16}{|l|}{ General preférences } \\
\hline Input Requirements & \multicolumn{3}{|c|}{ fullKeyboardControl } & \multicolumn{3}{|c|}{ fullkeyboardControl } & \multicolumn{3}{|c|}{ no recommendation } & \multicolumn{3}{|c|}{ fullkeyboardControl } & \multicolumn{3}{|c|}{ fullKeyboardControl, fullMouseControl } \\
\hline Hazard Type Avoldance & \multicolumn{3}{|c|}{ flassing, sound, motionsimulation } & \multicolumn{3}{|c|}{ flasshing, sound, motionsimulation } & \multicolumn{3}{|l|}{ sound } & \multicolumn{3}{|c|}{ flasshing, sound, motionsimulation } & \multicolumn{3}{|c|}{ motionsimulation } \\
\hline $\begin{array}{l}\text { Interoperability to Assistive } \\
\text { Technology }\end{array}$ & \multicolumn{3}{|l|}{$\begin{array}{l}\text { Ve TRUE } \\
\end{array}$} & \multicolumn{3}{|l|}{ TRUE } & \multicolumn{3}{|l|}{ TRUE } & \multicolumn{3}{|l|}{ TRUE } & \multicolumn{3}{|l|}{ TRUE } \\
\hline $\begin{array}{l}\text { Educational Complexity of } \\
\text { Adaptation }\end{array}$ & \multicolumn{3}{|c|}{ simplified or enriched } & \multicolumn{3}{|c|}{ simplified or enriched } & \multicolumn{3}{|c|}{ simplified or enriched } & \multicolumn{3}{|l|}{ simplified } & \multicolumn{3}{|c|}{ simplified or enriched } \\
\hline $\begin{array}{l}\text { Educational Level of } \\
\text { Adaptation }\end{array}$ & \multicolumn{3}{|l|}{ string } & \multicolumn{3}{|l|}{ string } & \multicolumn{3}{|l|}{ string } & \multicolumn{3}{|l|}{ string } & \multicolumn{3}{|l|}{ string } \\
\hline Language of Adaptation & \multicolumn{3}{|l|}{ string } & string & & & string & & & string & & & string & & \\
\hline Digital resource pereferences & & & & & & & & & & & & & & & \\
\hline Original Access Mode & \begin{tabular}{|c|c|} 
Required Access \\
Mode
\end{tabular} & $\begin{array}{c}\text { Required Adaptation } \\
\text { Type }\end{array}$ & $\begin{array}{c}\text { Required Adaptation } \\
\text { Detail }\end{array}$ & \begin{tabular}{|c} 
Required Access \\
Mode
\end{tabular} & $\begin{array}{c}\text { Required Adaptation } \\
\text { Type }\end{array}$ & \begin{tabular}{|c||}
$\begin{array}{c}\text { Required Adaptation } \\
\text { Detall }\end{array}$ \\
|
\end{tabular} & $\begin{array}{c}\text { Required Access } \\
\text { Mode }\end{array}$ & 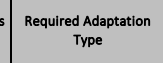 & $\begin{array}{l}\text { Required Adapotation } \\
\text { Detail }\end{array}$ & $\begin{array}{c}\text { Required Access } \\
\text { Mode }\end{array}$ & $\begin{array}{c}\text { Required Adaptation } \\
\text { Type }\end{array}$ & $\begin{array}{c}\text { Required Adaptation } \\
\text { Detall }\end{array}$ & \begin{tabular}{|c|c|c|} 
Required Access \\
Mode
\end{tabular} & $\begin{array}{c}\text { Required Adaptation } \\
\text { Type }\end{array}$ & $\begin{array}{c}\text { Required Adaptation } \\
\text { Detail }\end{array}$ \\
\hline auditory & textual, auditory & \begin{tabular}{|l|} 
transcript, \\
LongDescription
\end{tabular} & verbatim & textual, auditory & \begin{tabular}{|l}
$\begin{array}{l}\text { LongDescription, } \\
\text { transcript }\end{array}$ \\
\end{tabular} & verbatim & textual, visual & \begin{tabular}{|l} 
signlanguage, \\
transcript, caption
\end{tabular} & enhanced, verbatim & $\begin{array}{l}\text { auditory, visual, } \\
\text { textual }\end{array}$ & transcript & \begin{tabular}{|l||} 
enhanced, \\
synthesized, symbolic
\end{tabular} & 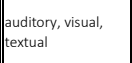 & transcript & \begin{tabular}{|l}
$\begin{array}{l}\text { enhanced, symbolic, } \\
\text { synthesized }\end{array}$ \\
\end{tabular} \\
\hline colour & textual, auditory & atternative Text & no recommendations & |isual, textual & \begin{tabular}{|l}
$\begin{array}{l}\text { highcontrast, } \\
\text { alternative Text }\end{array}$ \\
\end{tabular} & enhanced & no change & no recommendations & no recommendations & visual & \begin{tabular}{|l} 
highcontrast, \\
alternative Text
\end{tabular} & enhanced & no change & no recommendations & no recommendations \\
\hline itemsize & no change & no recommendations & no recommendations & visual & [Zooming] & enhanced & no change & no recommendations & no recommendations & |isual & [Zooming] & enhanced & no change & no recommendations, & no recommendations \\
\hline orientation & textual, auditory & \begin{tabular}{|l} 
alternativeText, \\
longDescription
\end{tabular} & $\begin{array}{l}\text { recorded, enhanced, } \\
\text { synthesized }\end{array}$ & $\begin{array}{l}\text { visual, textual, } \\
\text { auditory } \\
\end{array}$ & \begin{tabular}{|l|} 
alternativive ext, \\
longnescription \\
\end{tabular} & $\begin{array}{l}\text { recorded, enhanced, } \\
\text { synthesized }\end{array}$ & no change & no recommendations & no recommendations & \begin{tabular}{|l} 
visual, auditory, \\
textual
\end{tabular} & alternative Text & \begin{tabular}{|l|}
$\begin{array}{l}\text { recorded, enhanced, } \\
\text { synthesized }\end{array}$ \\
\end{tabular} & |isual & no recommendations & enhanced, symbolic \\
\hline posstion & textual, auditory & $\begin{array}{l}\text { alternative Text, } \\
\text { longDescription }\end{array}$ & $\begin{array}{l}\text { recorded, enhanced, } \\
\text { synthesized }\end{array}$ & \begin{tabular}{|l} 
visual, textual, \\
auditory
\end{tabular} & $\begin{array}{l}\text { alternative Text, } \\
\text { longeescription }\end{array}$ & $\begin{array}{l}\text { recorded, enhanced, } \\
\text { synthesized }\end{array}$ & no change & no recommendations & no recommendations & $\begin{array}{l}\text { Wisual, auditory, } \\
\text { textual }\end{array}$ & alternative $T$ ext & \begin{tabular}{|l||} 
recorded, enhanced, \\
synthesized
\end{tabular} & |isual & no recommendations & enhanced, symbolic \\
\hline textonlmage & textual, auditory & \begin{tabular}{|l|} 
alternativeText, \\
longDescription
\end{tabular} & \begin{tabular}{|l}
$\begin{array}{l}\text { recorded, enhanced, } \\
\text { synthesized }\end{array}$ \\
\end{tabular} & \begin{tabular}{|l|} 
visual, textual, \\
auditory \\
\end{tabular} & \begin{tabular}{|l}
$\begin{array}{l}\text { highContrast, } \\
\text { alternativerext or } \\
\text { longDescription }\end{array}$ \\
\end{tabular} & \begin{tabular}{|l} 
recorded, enhanced, \\
synthesized
\end{tabular} & |isisual & signlanguage & enhanced & 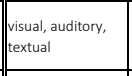 & $\begin{array}{l}\text { highcontrast, } \\
\text { alternative Text }\end{array}$ & \begin{tabular}{|l||}
$\begin{array}{l}\text { recorded, enhanced, } \\
\text { synthesized }\end{array}$ \\
\end{tabular} & |visual, textual & no recommendations : & symbolic \\
\hline textual & textual & no recommendations & no recommendations & |visual & [Zooming], [CSS] & as is & textual, visual & signlanguage & no recommendations & textual or visual & [CSS), [Zooming] & symbolic & |visual, textual & no recommendations : & $\begin{array}{l}\text { recorded, enhanced, } \\
\text { symbolic }\end{array}$ \\
\hline visual & textual, auditory & $\begin{array}{l}\text { alternative Text, } \\
\text { transcript, e-book, } \\
\text { audioDescription }\end{array}$ & $\begin{array}{l}\text { enhanced, synthesized, } \\
\text { realtime, recorded }\end{array}$ & $\begin{array}{l}\text { visual, textual, } \\
\text { auditory }\end{array}$ & \begin{tabular}{|l} 
highcontrast, \\
alternativeText, \\
transcript, e-book, \\
audioDescription \\
\end{tabular} & $\begin{array}{l}\text { enhanced, synthesized., } \\
\text { reattime, recorded }\end{array}$ & visual, textual & \begin{tabular}{|l}
$\begin{array}{l}\text { captions, } \\
\text { signtLanguage, } \\
\text { alternative Text, } \\
\text { longDescription }\end{array}$ \\
\end{tabular} & $\begin{array}{l}\text { enhanced, verbatim, } \\
\text { realtime, }\end{array}$ & $\begin{array}{l}\text { visual, textual, } \\
\text { auditory }\end{array}$ & $\begin{array}{l}\text { alternative Text, } \\
\text { audioDescription, } \\
\text { highhontrast, } \\
\text { transcript. }\end{array}$ & \begin{tabular}{|l||}
$\begin{array}{l}\text { enhanced, } \\
\text { synthesized, reattime, } \\
\text { recorded, symbolic }\end{array}$ \\
\end{tabular} & |visual, textual & dations :" & $\begin{array}{l}\text { recorded, enhanced, } \\
\text { symbolic }\end{array}$ \\
\hline
\end{tabular}

Figure 2. Learner input profile. (The images in the table are taken from https://icons8.com under the Creative Commons Attribution-NoDerivs 3.0 Unported license). 


\section{AccessibleOCW Ontology}

Our AccessibleOCW ontology is currently composed of 16 classes. It is is available and deployed in a VoCol [11] environment (http:/ / vocol.iais.fraunhofer.de/accessibilityOnto/) (a visual representation of the ontology, created with WebVOWL [12], is shown in Figure A1 in Appendix A of this article). It contains two main classes, as introduced in Section 2.1, Learners and Digital Resources, with their relevant properties and other classes that are used to describe the different representations of the educational resources and preferences of the learners [3]. The ontology had been revised and extended since we introduced it in our previous work. In the latest version, we added the profiles of five types of disabilities and added, removed, and edited several relationships, accordingly.

The Learner class specifies the accessibility needs of learners by reusing the User concept of the ACCESSIBLE ontology, to represent the domain knowledge of disability types, characteristics, functional limitations, and assistive technologies, whereas the accessibility needs of e-learning are represented by the IMS PNP specifications [8]. The properties of the Learner class, as listed in Figure 2, include the required input control (keyboard, mouse, etc.), the language, the level of education, the usage of assistive technology, the recommended complexity level of the displayed materials (e.g., simplified, enriched, or complex material), and any hazard that should be avoided (e.g., flashing, sound, or motion simulation). In conjunction with these properties, other classes are used to define the learner's preferences of the learning resources and recommended adaptations, as illustrated in Figure A1 in Appendix A. For example, if we have a video resource, a textual alternative might be requested by a blind person, and this might be in the form of a transcript of this video or another alternative readable document (e.g., an e-book). As the Learner class extends the User class from the ACCESSIBLE ontology, SWRL rules can be used to infer properties; such as the one given in Listing 1 , which describes a rule to infer the isAtInteroperable property from certain ACCESSIBLE ontology concepts. It states that if a user has a disability and this disability type requires an assistive device, then the property isAtInteroperable is considered to be true.

Listing 1. SWRL rule for inferring the property of assistive technology interoperability.

User_has_Disability (?x,?y) -Disability_has_Device (?y,?z)

->isAtInteroperable (?x,true)

The Digital Resource class refers to the type of educational material representation (e.g., video, slide presentation, or text document) and is defined in terms of the IMS AfA Digital Resource Description (DRD) specification [9]. A digital resource has several properties, such as the representation type of the resource (AccessMode), the language of representation, and references to other language adaptations if any, as well as the complexity type of the representation (e.g., simplified, enriched, or complex). If the digital resource has another alternative adaptation, the type and references are included, and if a resource is an adaptation of another resource, this is also defined in these properties; a resource might be a full adaptation or a partial adaptation of another resource. More properties related to the adaptation of special items in a resource (e.g., font size) are also included, which are helpful when performing CSS adaptations. The standards that the resource follows and its accessibility to APIs and assistive technologies, as well as the flexibility of control with mouse and keyboard access, are included as well. Some properties were not considered in our ontology, as they are out of scope of today's OCW systems, such as tactile, which is related to the sense of touch, and olfactory, which is related the sense of smell. 


\section{Proposed Learner Profiles}

We created five examples of learner profiles to describe different needs and preferences of learners based on the AccessibleOCW ontology. Each profile represents a type of disability with its recommended preferences, which are used as guidelines for the learner's input profile in Section 5 .

The rationale behind creating these profiles is to provide a guided list of preferences for each type of learner, from which the learners can edit and input their actual profiles. These profiles were created by analyzing the features and properties of users defined in the following two resources: (i) GPII [6], an infrastructure proposed by an international consortium to provide auto-personalization for different types of device interfaces; and (ii) the accessibility needs of learners in massive open online courses (MOOCs) [13]. Both resources use WCAG2.0 guidelines to categorize and define the types of user needs and preferences.

Each profile is defined as an instance of the Learner class with some recommended properties as per the type of learner. For example, Listing 2 provides a representation of the blind learner profile in our ontology.

Listing 2. Example representation of a blind learner.

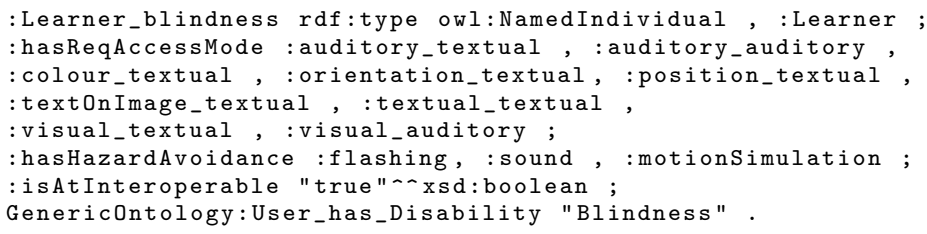

The five profiles, together with their properties and recommended digital resource adaptations, are defined in Figure 2. The terms used in the table are the classes and properties defined in our ontology, which have been adopted from the data element specification of IMS AfA [5], as explained in Section 3. The upper part of the table defines the learner's properties, including the input requirements, the hazard interface that should be avoided, assistive technology usage, the educational level of adaptation, the language, and the level of education, which are string values left to the learner's selection. The lower part of the table defines the recommended adaptations for the digital resources, where it includes the original type of resources and the adaptation requirement for each type of user, with details on how to implement those adaptations (alt-text, caption, transcript, etc.). For some preferences, we are using "no recommendation" or "no change", as sometimes there is no specific recommendation or these properties are not relevant to the learner; for example, the size of objects (itemsize) is not of relevance to a blind learner. The five profiles are categorized and briefly described as follows:

1. Blindness. This category includes totally blind users, where text cannot be read by any means of magnification. In this category, learners require full control keyboards as an input device. Content with flashing sound and motion simulation should be avoided. Assistive technologies are used, such as screen and Braille readers. The language and complexity preferences of the displayed material depends on the learner's preferred language and level of education. In terms of the digital resource recommendations, learners would always require an alternative textual or auditory description for any visual or audio representation (alternative text for image, transcript for audio files, etc.).

2. Low Vision. This category includes users with low sight and color-blind users. Learners with low vision share several preferences with blind learners, but they also require zooming for text and visual objects, which means that a digital resource should allow zooming while preserving the content structure. 
3. Deaf and Hard of Hearing. This category includes users with no or too little hearing. A learner might require sign language instead of textual materials.

4. Cognitive, Language, and Learning Disabilities. This category includes users with low literacy. Learners with cognitive impairments often prefer simplified versions of educational material and might also require special formatting of text (i.e., specific CSS styling).

5. Physical Disabilities. This category includes users with physical function limitations, such as general mobility or the moving of hands and arms. Some learners with physical impairments might require full mouse control.

\section{Personalization Module}

The personalization module is composed of a learner profile, where the learners select and create their preferences and then save their profiles, and a matching approach to map learners to appropriate educational resources. In the following, we describe the implementation of the learner input profile. We also define an example query for retrieving educational resources with respect to the learner's input profile but without implementation of the interface; this is considered for future work.

We implemented a web form to collect and save the learners' profiles. The learners should first select the type of their profiles from our five proposed profiles, as illustrated in Figure 3. Then, the web form, as illustrated in Figure 4, is loaded with the initial preferences that match the learner's type selection as per the ontology definitions. The user input is saved in an RDF file, which is compliant to the specifications of IMS PNP in order to use it for mapping to the properties of the educational resources represented in the IMS DRD format. In the future, we envision the learner profile to be saved in a data store, which follows the AccessibleOCW schema in order to access its values and map them to the educational resources.

The web form is implemented using the JavaScript library React [14]. We opted for React as it already supports several accessibility concepts, such as ARIA attributes, and as it is widely used in web development. It furthermore supports the development of websites as separate components. This component structure can be useful when adapting and extending the web interface. We used SPARQL [15] to query the ontology, based on Fuseki as SPARQL server [16]. We created a SPARQL client in React that accesses our ontology on the Fuseki server.

SPARQL queries are used to load the relevant preferences (input requirement, type of preferred resources, etc.) of the leaner's profile from the AccessibleOCW ontology. For example, Listing 3 shows a SPARQL query that retrieves the properties of a blind user profile. The learners then create their personal profiles by editing their preferences and saving their profiles. The resulting profile can be used as an individual account or an account for a group of users, since in some cases learners might have difficulties entering preferences by themselves.

Listing 3. SPARQL query for retrieving the properties for the blind learner profile.

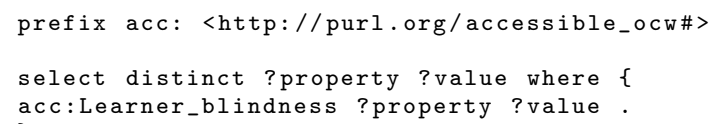

Based on the profile information, the OCW content can be adapted to the individual needs and preferences of the learners, and the most appropriate educational resources can be suggested to them. For example, the SPARQL query in Listing 4 is used to retrieve the recommended digital resources with respect to the accessibility preferences of a blind user. 


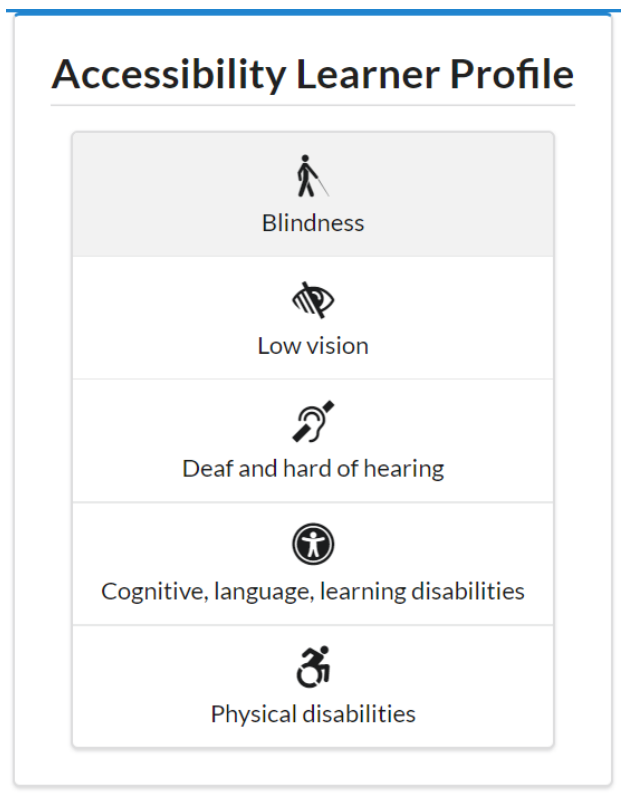

Figure 3. GUI to select and configure the accessibility learner profile.

\section{Accessibility Learner Profile}

This profile is focusing on the preferences of users with accessibility needs. The profile can be used for individual account or by a group of users

\begin{tabular}{|c|c|c|c|}
\hline \multicolumn{4}{|l|}{ Personal Information } \\
\hline \multicolumn{4}{|l|}{ Profile Name } \\
\hline \multicolumn{4}{|l|}{ Profile Name } \\
\hline Language & & Educational Level & \\
\hline Dutch & $\checkmark$ & Bachelor Degree & $\checkmark$ \\
\hline Input Requirement & & Assistive Technology & \\
\hline Full Keyboard Control & $\checkmark$ & Screen Reader & $\checkmark$ \\
\hline
\end{tabular}

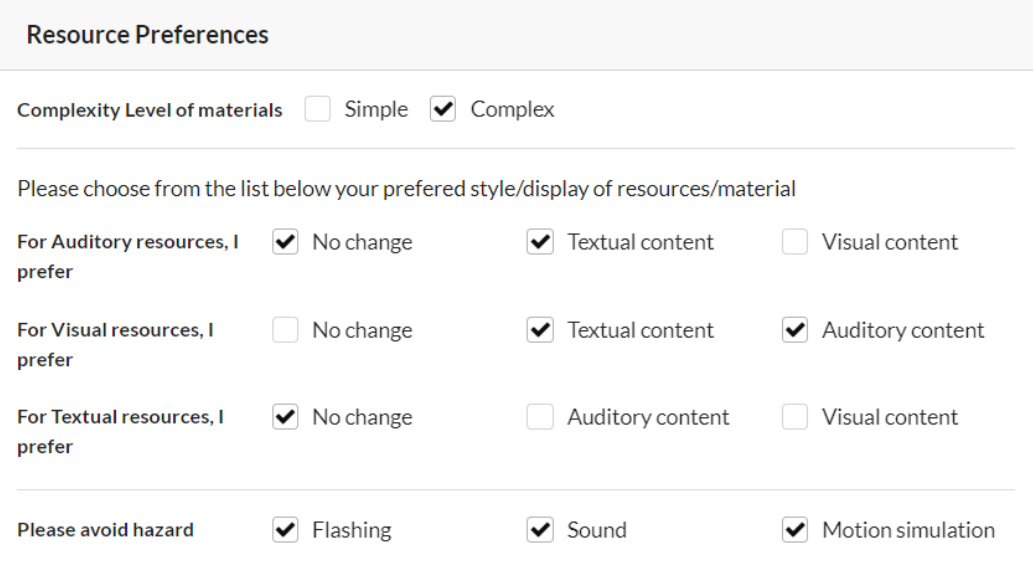

Figure 4. Accessibility learner profile form. 
Listing 4. SPARQL query: Selecting the relevant educational resources.

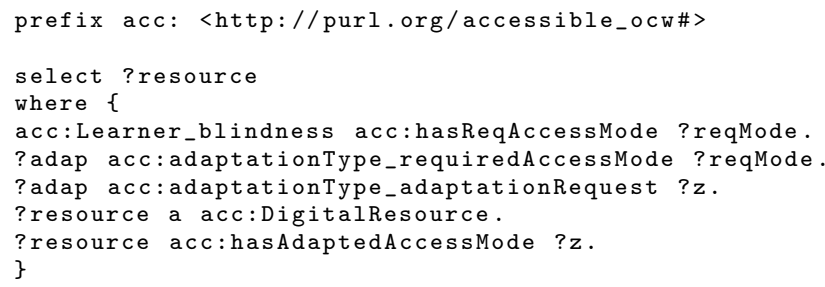

\section{Evaluation}

We defined five example profiles for learners with different types of disabilities. In order to evaluate these profiles, we designed a questionnaire and collected the preferences and needs of different types of learners. The main idea of the questionnaire is to validate the preferences which were created for each type of disability, and to make sure that the recommended preferences match the learner needs. The questionnaire is composed of five questions with a list of answers to select from (allowing for multiple answers per question). Participants were also encouraged to make comments and provide feedback.

1. What would the learner prefer as an input requirement? (i) full keyboard control; (ii) full mouse control

2. What hazard content would the learner prefer to avoid? (i) flashing; (ii) sound; (iii) motion simulation

3. What would the learner prefer educational material to contain? (i) simplified content; (ii) enriched content; (iii) complex content

4. Would the learner use assistive technologies? (i) yes; (ii) no; (iii) maybe

5. If the educational material makes use of text, color, audio, position, text on images, item size, or visual content, which type of alternative would the learner prefer? (i) no alternative; (ii) text alternative; (iii) audio alternative; (iv) visual alternative.

We collected the feedback of seven experts; four of them are working with blind and low-vision learners, one of them is working with learners with cognitive impairments (in particular, intellectual and neurodevelopmental disabilities), and two experts are in the usability and web accessibility field. We obtained sufficient feedback for the blind and low-vision profiles, but not enough feedback for the other profiles, especially for the deaf and physically impairment profiles, in terms of validating them and aligning our ontology. However, the feedback and comments we received are valuable and help in shaping our future work. The preferences that were defined for the blind and low-vision profiles reveal the learner's needs as per the questionnaire answers. However, there might be personal preferences for some properties, such as preferring auditory or textual alternatives for describing charts and colors. The web form is designed to address these variations of preferences, based on the predefined profiles that provide general guidelines the learner can edit and adapt.

Important feedback has addressed the privacy and discrimination of learners. The idea of asking learners to select their type of disability before filling their learner profile violates the privacy of learners. One alternative would be to detect the needs and preferences from how particular learners use their browser (e.g., detecting screen reader usage) and adapt the system with respect to it, or to allow them to select their preferences from a general list of properties that are not related to a special type of disability or group of users. Accordingly, the five example profiles may not be used for loading the field values in the web form, as per the learner disability selection, but can be used in the back-end to provide matches between the learner preferences and educational resources as well as alternatives. These profiles can be used in the OCW authoring tool to guide authors when they are 
creating educational material, especially when they are targeting special types of learners. For example, if an author is creating educational material for learners with learning disabilities, a validation can be added in the authoring tool that evaluates the complexity of the text and the format of the designed material with respect to this type of learner.

\section{Related Work}

In our previous work [2], we reviewed and classified the state of the art, and identified the most relevant standards related to web accessibility in e-learning contexts. We analyzed 11 web accessibility standards, guidelines, and techniques, and examined 20 ontologies that are available in the literature and on the web. As a result of that survey, we defined a set of OCW accessibility needs and requirements, and identified standards and ontologies that best meet our requirements. We also had a look at related ontologies that were developed specifically for e-learning contexts.

The Learning Object Context Ontologies (LOCOs) are a group of ontologies developed to ease the exchange of data between multiple educational services [17]. Among the LOCOs, one ontology is addressing the learner preferences in accordance with the IMS Learner Information Package (LIP) specification (e.g., affiliations, goals, identifications, interests, and qualifications) [18], but without addressing the accessibility needs of learners. Another related ontology is ADOOLES (Ability and Disability Ontology for Online Learning and Services), which has been developed to annotate learning resources [19]. It is based on the ADOLENA ontology [20], which has been used to enhance search capabilities by Ontology-Based Data Access (OBDA). ADOOLES represents knowledge in the domain of e-learning and includes a set of concepts describing disabilities. However, the number and types of disabilities covered by ADOOLES are very limited, and organized as a simple class hierarchy without any properties and further linking.

A number of approaches have been proposed to represent accessibility knowledge and requirements through the definition of learner profiles and educational resources. A preliminary proposal of using the IMS AfA specifications to describe the preferences of learners in MOOCs has been presented by Iniesto \& Rodrigo [21]. It makes use of accessibility metadata to feed a recommendation system, which is related to our work. However, the authors only specified a list of metadata from the IMS AfA, which they plan to use to define the learner preferences, but they did not come up with a formal representation of the metadata or any way to input the learner profile into a system. Moreover, they do not consider the relationships between learner preferences and digital resource properties, as we did in our work.

\section{Conclusions}

In this article, we presented the AccessibleOCW ontology to represent the accessibility needs of learners in OCW systems with respect to the IMS AfA specifications. The ontology definitions, together with rule-based queries, can be used to retrieve relevant educational resources. In addition, we proposed five learner profiles to represent different accessibility needs and preferences of learners in OCW systems, using our AccessibleOCW ontology. We implemented a web form that represents these preferences in order to facilitate the creation of learner profiles. The profiles are used to select the educational resources and their alternatives as per the learner's defined preferences. We conducted a questionnaire-based evaluation to collect the preferences of each type of disability for the sake of evaluating the five proposed learner profiles and collecting feedback regarding the learner profile representation. For future work, we are planning to use the learner profiles for suggesting alternative representations of educational resources in authoring tools, where the authors can receive suggestions for creating more accessible educational materials that can be used by a wider range of learners.

Author Contributions: Conceptualization, M.E. and S.L.; Methodology, M.E and S.L.; Software, M.E.; Validation, M.E. and S.L.; Data curation, M.E., S.L, ; Writing—original draft preparation, M.E. and S.L.; writing-review and editing, M.E., S.L. and S.A.; Visualization, M.E., S.L.; Supervision, S.L. and S.A.; Project administration, S.L.; Funding acquisition, S.L. and S.A. 
Funding: This research was funded by the EU project SlideWiki (grant no. 688095) and by the BMBF project InclusiveOCW (grant no. 01PE17004D).

Acknowledgments: We would like to thank all experts who participated in the evaluation.

Conflicts of Interest: The authors declare no conflict of interest.

\section{Appendix A. AccessibleOCW Ontology}

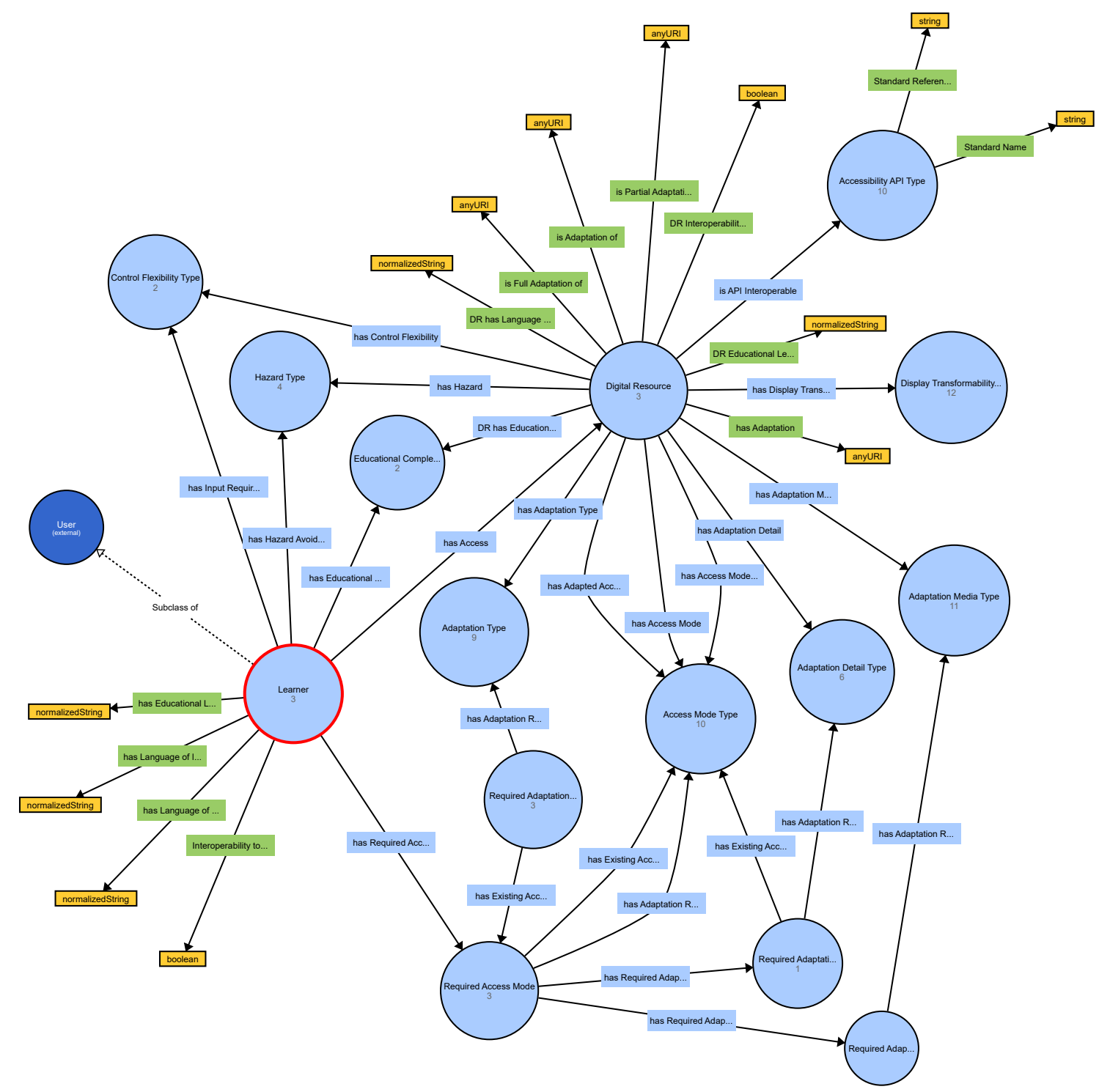

Figure A1. Visual representation of our AccessibleOCW ontology, created with the tool WebVOWL [12].

\section{References}

1. Open Education Consortium: What is Open Courseware? Available online: http:/ /www.oeconsortium.org/ faq/what-is-open-courseware/ (accessed on 28 November 2018).

2. Elias, M.; Lohmann, S.; Auer, S. Fostering Accessibility for OpenCourseWare with Semantic Technologies-A Literature Review. In Proceedings of the 7th International Conference on Knowledge Engineering and Semantic Web (KESW '16), Prague, Czech Republic, 21-23 September 2016; Springer: Cham, Swithzerland, 2016; pp. 241-256. 
3. Elias, M.; Lohmann, S.; Auer, S. Ontology-Based Representation of Learner Profiles for Accessible OpenCourseWare Systems. In Proceedings of the International Conference on Knowledge Engineering and the Semantic Web (KESW), Szczecin, Poland, 8-10 November 2017; Springer: Cham, Swithzerland, 2017; pp. 279-294.

4. Grammati-Eirini, K.; Lopes, R. Deliverable 4.1-A Set of Formalisms and Taxonomies for Accessibility Assessment Procedures and Their Inherent Meta Models; Technical Report, ACCESSIBLE (Grant Agreement No. 224145); ACCESSIBLE: Thessaloniki, Greece, 2009.

5. IMS Access For All. Available online: https://www.imsglobal.org/activity/accessibility (accessed on 28 November 2018).

6. Global Public Inclusive Infrastructure GPII. Available online: https://ds.gpii.net/learn/accessibilitymasterlist (accessed on 28 November 2018).

7. World Health Organization. International Classification of Functioning, Disability and Health (ICF); WHO: Geneva, Swithzerland, 2001.

8. IMS Personal Needs\& Preferences (PNP) Specification Information Model. Available online: https:/ / www.imsglobal.org/accessibility/afav3p0pd/AfA3p0_PNPinfoModel_v1p0pd.html (accessed on 28 November 2018).

9. IMS Digital Resource Description Specification Information Model. Available online: http:/ /www.imsglobal. org/accessibility /afav3p0pd/AfA3p0_DRDinfoModel_v1p0pd.html (accessed on 28 November 2018).

10. Project, A.M. Making Accessible Content Discoverable: A Benetech Led Proposal for Accessibility Metadata in Schema.org. 2014. Available online: http:/ / www.a11ymetadata.org/ (accessed on 28 November 2018).

11. Halilaj, L.; Petersen, N.; Grangel-González, I.; Lange, C.; Auer, S.; Coskun, G.; Lohmann, S. VoCol: An Integrated Environment to Support Version-Controlled Vocabulary Development. In Proceedings of the 20th International Conference on Knowledge Engineering and Knowledge Management (EKAW 2016), Bologna, Italy, 19-23 November 2016; Springer: Cham, Swithzerland, 2016; pp. 303-319.

12. Lohmann, S.; Link, V.; Marbach, E.; Negru, S. WebVOWL: Web-based Visualization of Ontologies. In Proceedings of the EKAW 2014 Satellite Events, Linköping, Sweden, 24-28 November 2014; Springer: Cham, Swithzerland, 2015; Volume 8982, pp. 154-158.

13. Sanchez-Gordon, S.; Luján-Mora, S. Web Accessibility Requirements for Massive Open Online Courses. Can MOOCs be really universal and open to anyone? In Proceedings of the V Congreso Internacional sobre Calidad y Accesibilidad de la Formación Virtual (CAFVIR 2014), Antigua Guatemala, Guatemala, 14-16 May 2014.

14. React (JavaScript library). Available online: https:/ / facebook.github.io/ react/ (accessed on 28 November 2018).

15. SPARQL Query Language. Available online: https://www.w3.org/TR/rdf-sparql-query/ (accessed on 28 November 2018).

16. Apache Jena Fuseki. Available online: https://jena.apache.org/documentation/fuseki2/ (accessed on 28 November 2018).

17. Jovanović, J.; Gašević, D.; Devedžić, V. Dynamic assembly of personalized learning content on the semantic web. In Proceedings of the 3rd European Semantic Web Conference (ESWC'06), Budva, Montenegro, 11-14 June 2006; Springer: Berlin/Heidelberg, Germany, 2006; pp. 545-559.

18. IMS Learner Information Package Specification. Available online: http:/ /www.imsglobal.org/profiles / index.html (accessed on 28 November 2018).

19. Nganji, J.T.; Brayshaw, M.; Tompsett, B. Ontology-driven disability-aware e-learning personalisation with ONTODAPS. Campus Wide Inf. Syst. 2012, 30, 17-34. [CrossRef]

20. Maria, K.C.; Ronell, A.; Aurona,G.; Gibson, C. Enhancing Web Portals with Ontology-Based Data Access: The Case Study of South Africa's Accessibility Portal for People with Disabilities. In Proceedings of the OWL: Experiences and Direction (OWLED '08), Karlsruhe, Germany, 26-27 October 2008; Volume 432.

21. Iniesto, F.; Rodrigo, C. Accessible user profile modeling for academic services based on MOOCs. In Proceedings of the XVI International Conference on Human Computer Interaction, Vilanova i la Geltru, Spain, 7-9 September 2015; ACM: New York, NY, USA, 2015; p. 55.

(C) 2018 by the authors. Licensee MDPI, Basel, Switzerland. This article is an open access article distributed under the terms and conditions of the Creative Commons Attribution (CC BY) license (http://creativecommons.org/licenses/by/4.0/). 